\title{
Development of a direct fabrication technique for full-shell x-ray optics
} M. Gubarevํㅣ J.K.Kolodziejczak ${ }^{1}$, C. Griffith, J. Roche, W. S. Smith ${ }^{1}$, T. Kester ${ }^{1}$, C. Atkins², W. Arnold $^{3}$ and B. Ramsey ${ }^{1}$

\author{
${ }^{1}$ NASA Marshall Space Flight Center, Huntsville, AL, USA \\ ${ }^{2}$ University of Alabama at Huntsville, Huntsville, AL, USA \\ ${ }^{3}$ A. I. Solutions, Inc., Huntsville, AL, USA
}

\begin{abstract}
Future astrophysical missions will require fabrication technology capable of producing high angular resolution x-ray optics. A full-shell direct fabrication approach using modern robotic polishing machines has the potential for producing high resolution, light-weight and affordable x-ray mirrors that can be nested to produce large collecting area. This approach to mirror fabrication, based on the use of the metal substrates coated with nickel phosphorous alloy, is being pursued at MSFC. The design of the polishing fixtures for the direct fabrication, the surface figure metrology techniques used and the results of the polishing experiments are presented.
\end{abstract}

Keywords: x-ray optics, full-shell x-ray optics, direct fabrication

\section{INTRODUCTION}

$\mathrm{X}$-ray optics envisioned for future astrophysics missions would have angular resolutions comparable to that of the Chandra observatory and provide at least an order of magnitude larger effective area. The AXAF (Advanced X-ray Astrophysics Facility, now Chandra) program used a "classical" approach wherein a surface profile with the required optical prescription was ground and polished directly into a full shell substrate. The Chandra mirrors were relatively thick (20-25 mm) to provide adequate support for the mirror during fabrication, metrology and assembly so the integrity and the optical performance of the mirror was preserved. An alternative approach, that of replication, developed both for fabrication of full shell and segmented mirrors [1,2] aims to decrease the mirror thickness to a fraction of millimeter so dense nesting of light-weighted mirrors would lead to increased effective area of the telescope. However, the low stiffness of these thinner mirrors makes them more susceptible to fabrication-induced figure errors and to mounting distortions, all of which result in angular resolutions over an order of magnitude worse than Chandra. The challenge for modern $\mathrm{x}$-ray optics fabrication techniques is to preserve the angular resolution that can be achieved by the use of the Chandra-like direct polishing techniques but with much thinner substrates.

Recently, two direct fabrication techniques have been under development for light-weight x-ray optics. Preliminary results demonstrate the potential for the technique to reach the angular resolution of 1-2 arc seconds HPD and better. In both techniques the desired surface profile is polished directly onto substrate. One of the techniques under development at Goddard Space Flight Center uses segmented substrates made from crystalline silicon [3], the other utilizes the full-shell approach. The latter utilizes substrates from fused silica (Astronomical Observatory of Brera, Italy) [4, 5] or metal [6] (Marshall Space Flight Center.) This approach capitalizes on modern methods of figuring and polishing the mirrors with low surface errors but requires thicker substrates than mirrors fabricated from replication techniques. We report here the status of the direct fabrication technology development at Marshall Space Flight Center (MSFC).

Development of the direct fabrication system will permit the demonstration of high resolution optics made from lightweight materials that will satisfy the needs of future x-ray astronomy missions. These metal optics are well suited for a wide variety of applications from future small explorers through to probe-class missions. Direct fabrication technology

*Mikhail.V.Gubarev@nasa.gov; Phone: 1256 544-7816; FAX 1256 544-2659 
could also be utilized for fabrication of high resolution optics for the X-ray Surveyor [7] both as full-shell optics for inner mirrors and as segmented optics for outer mirrors of the telescope.

The use of metal substrates permits utilizing single-point diamond turning, which minimizes subsurface damage compared with grinding, so less time needs to be used to remove the damage. After diamond turning, the mirrors would be heat treated to relieve residual stresses, machined to the final form and thickness, and then plated with electroless nickel. In order to compensate for plating stresses the plating will be done in two stages; First the minimum thickness will be plated on the back surface with the front surface masked, then the rear surface will be masked and the front (mirror) surface will be plated thicker material to account for optical processing. Now the nickel mirror surface is single-point diamond turned again. The result will be a surface with a 1-to-2 micrometer surface error and a few 10's of nm surface finish. The next step will be the deterministic computer controlled polishing. The MSFC Zeeko IRP600X 7-axis CNC polishing machine [8] is used for this next step. The machine utilizes a "bonnet" technique in which an inflated rubber hemispherical diaphragm supports the polishing medium. The "bonnet" is attached to a spindle and compresses onto the work piece so that it conforms to the surface being polished. This permits a highly deterministic figuring of the surface. The bonnet rotates and moves across the surface to be polished, with the removal rate being dependent on both rotation and translation speed. Finally there are different "bonnet" sizes $(20 \mathrm{~mm}, 40 \mathrm{~mm}$ and $80 \mathrm{~mm}$ radii of curvature) so the footprint of the "bonnet" on the surface being polished can be varied over a wide range. This permits different spatial frequency surface errors to be attacked. This 7-axis computer-controlled polishing machine and the shape of bonnet polishing lap provides more precise control of the polishing parameters, such as the wear function rate, shape, and footprint, leading to a faster convergence compared to traditional polishing machines. The footprint will define the minimal size of the surface error features the machine is capable of removing, so the deterministic bonnet polishing would minimize the spatial frequency surface errors below some threshold frequency and the rest of the mid-frequency errors will be corrected using other techniques. For the Brera Observatory approach, an ion-figuring process can be used for further correction of the residual errors [5]. At MSFC, we are developing the differential deposition technique for correction of these mid-frequency spatial errors [9].

\section{DIRECT FABRICATION}

\subsection{Materials for direct fabrication technique}

Several metal alloys have been considered as a substrate for the directly fabricated x-ray mirrors. The candidates should have low density, low coefficient of thermal expansion (CTE), high modulus of elasticity and high yield strength. They can be coated with Nickel/Phosphorous (NiP) alloy, a hard material that can be easily figured and polished/superpolished,. NiP is the the coating used for all the mandrels that are fabricated at MSFC for its Electroformed Nickel Replication x-ray optics program. There is, therefore, considerable experience in machining and polishing this material. The properties of possible substrate materials are summarized in Table 1.

Table 1. Mechanical Properties of Potential Mirror Substrate Materials

\begin{tabular}{|c|c|c|c|c|}
\hline Material & $\begin{array}{c}\text { Density } \\
\left(\mathbf{g} / \mathbf{c m}^{\mathbf{3}}\right)\end{array}$ & $\begin{array}{c}\mathbf{C T E} \\
\left(\mathbf{1 0}^{-\mathbf{6}} / \mathbf{K}^{-\mathbf{1}}\right)\end{array}$ & $\begin{array}{c}\text { Elastic Modulus } \\
\mathbf{G P a}\end{array}$ & $\begin{array}{c}\text { Yield Strength } \\
\mathbf{M P a}\end{array}$ \\
\hline Beryllium & 1.8 & 12 & 318 & 240 \\
\hline $\mathrm{Al}(6061)$ & 2.7 & 24 & 69 & 276 \\
\hline $\mathrm{AlSi}$ & 2.8 & 17 & 90 & 235 \\
\hline BeAl-162Met & 2.1 & 13.9 & 193 & 314 \\
\hline $\begin{array}{c}\text { Duralcan F3S.30S } \\
\mathrm{AlSi}+\mathrm{SiC}(30 \% \text { by vol) }\end{array}$ & 2.8 & 14.6 & 120 & 210 \\
\hline
\end{tabular}

Beryllium and Beryllium-Aluminum alloys have excellent mechanical properties for an optic substrate. They have a very low density and a very high modulus, which makes for a stiff structure. The main drawback is beryllium's toxicity, which means that special facilities and controls are necessary during fabrication. However, beryllium and its alloys can be coated with NiP and numerous examples exist of beryllium optics being fabricated for NASA missions this 
way [10], [11]. The addition of Aluminum to Beryllium permits close matching of the alloy CTE to the CTE of NiP, improves machinability and also reduces the cost of the substrate material. General Dynamics of Cullman, AL (https://gdmissionsystems.com/imaging/services-solutions/beryllium-machining/) has extensive experience in fabrication of high-quality optical parts from Beryllium and its alloys and is experimenting with a low-temperature additive sintering technology that will produce a semi-porous and highly homogeneous sintered beryllium pre-form that is then infiltrated with an aluminum alloy. This results in low stress, low distortion, and low variability in reaching substrates of the desired sizes. Further, technique permits manufacturing of substrates for full-shell or segmented x-ray mirrors.

In addition to beryllium, other metal alloys are good candidates for optics substrates. A family of aluminum/silicon materials are used in aerospace and in the automobile industry - have low density, a CTE that can be tailored by varying the silicon content, and a high yield strength that approaches the ultimate tensile strength. The addition of silicon carbide provides further reduces the CTE, so it can match that of NiP. These materials can also be machined with near-standard processes. Large, high-quality components are routinely cast, by companies like Eck Industries, Inc. (http://www.eckindustries.com/), from this material making substrates readily available and relatively inexpensive. In addition, they can be coated with NiP [12], as with the beryllium.

An added bonus with this family of $\mathrm{BeAl}$ and AlSi materials is that the mirror-shell support structure could also be fabricated from it, simplifying the thermal design. Moreover the use of the metal alloys for the substrates permits fabrication of mounting flextures integral to the mirror for mounting the shell into the telescope structure, reducing the number of epoxy joints and thus producing more stable optics.

While the beryllium-based alloys offer the best properties, there are clearly multiple promising materials for light weight mirror fabrication. However, at present moment we are focusing on development of the technology to figure and polish thin x-ray metal optics directly. Hence, NiP-coated aluminum parts are used as a surrogate for development of most of the technological steps. This reduces costs considerably, makes for a much faster turnaround, and removes any precautions necessary to avoid accidentally exposing any underlying beryllium during processing at MSFC. In order to avoid issues associated with CTE mismatch between aluminum and NiP, the aluminum surrogates can be plated with NiP in such a way that the final thickness of the coating would be equal on the inner and outer surfaces of the shell, equalizing differential stresses induced by the CTE mismatch. Once the polishing techniques for production of full-shell metal optics are developed, they can be applied to the beryllium or aluminum-silicon alloys.

\subsection{Polishing experiments}

For thin-shell direct fabrication, one of the major challenges is to support the shell during diamond turning polishing and metrology so that, at one extreme, it cannot microyield, but also cannot deflect enough to affect the polishing itself. The design of the fixtures to support the shells during processing have already been described [6]. While the fixtures were in fabrication, efforts were concentrated on the development of polishing processes using the Zeeko polishing machine.
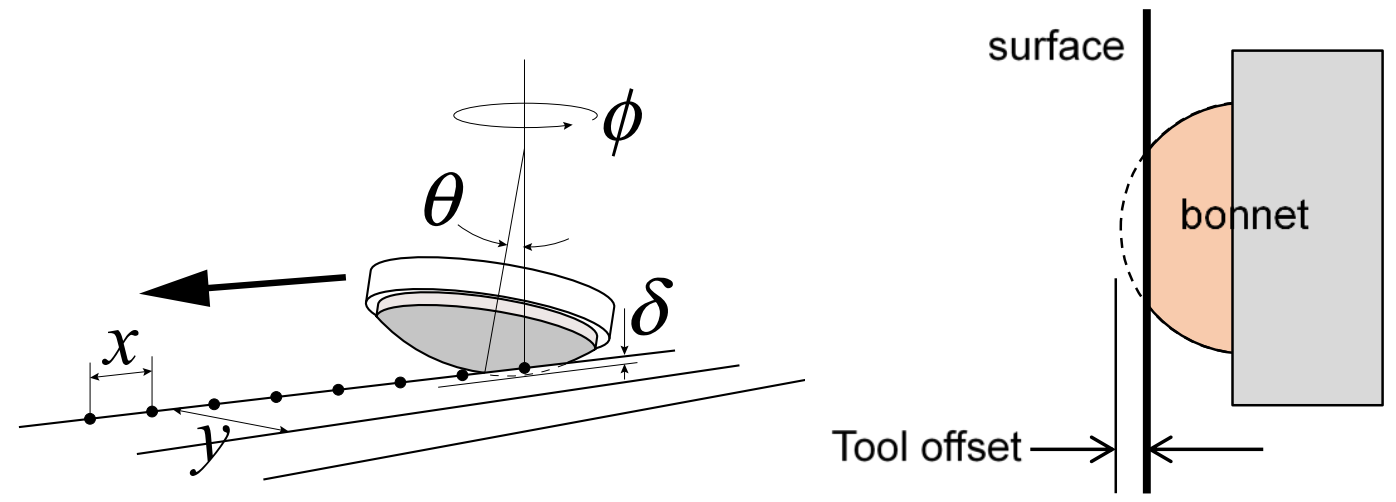

Figure 1. Illustration (left) of precess angle, $\theta$, phi angle, $\varphi$, tool offset, $\delta$, point spacing $\mathrm{x}$ and track spacing, y. Polishing bonnet moves at specified feed rate between points spaced at $\mathrm{x}$ along tracks spaced at $\mathrm{y}$. Figure at right shows tool offset. 
As the Zeeko machine comes with software intended for fabrication of normal-incidence optics, initial work started with development of custom tool-path generating (TPG) software optimized for the fabrication of high-resolution grazing-incidence mirrors. The objective was to determine the wear rate dependence on the machine setup parameters - tool feedrate, bonnet pressure, spindle rotation rate, tool offset, precess angle, and phi angle. Figure 1 illustrates the machine setup parameters. The dependence on bonnet characteristics (such as radius, thickness, and elastic modulus, as well as dependence on slurry characteristics such as particle grit size and solids fraction) also had to be determined. A model of the wear pattern as a function of numerous physical parameters was developed, so it can be calibrated with a relatively small set of direct measurements. The model was verified through series of the polishing experiments using $100 \mathrm{~mm}$ diameter NiP plated flat samples. The polishing parameters - feed rate, tool offset, etc. - were varied and the wear function dependence on these parameters was determined. The surface figure of the flat samples was measured using an optical

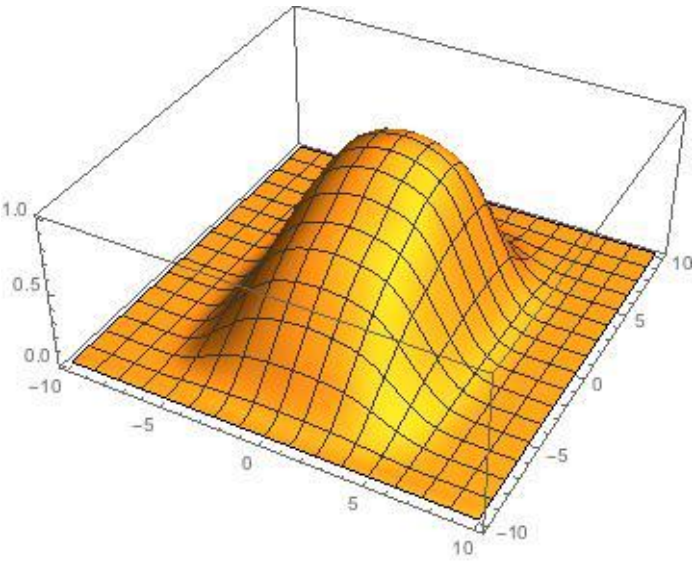

Figure 2. Example of machine wear function. The function is not symmetrical in the direction of the bonnet feed rate because the precess angle of 15 degrees. interferometer to produce a surface maps before and after a polishing runs. Example of the wear function is shown in Figure 2. The removal rate dependence on the tool offset was also measured. It was found that the feedrate flattens in the $0.7-0.8 \mathrm{~mm}$ offset range resulting in minimal sensitivity to alignment in a \pm 50 microns range around $0.75 \mathrm{~mm}$. This offset was used for all consecutive polishing experiments.

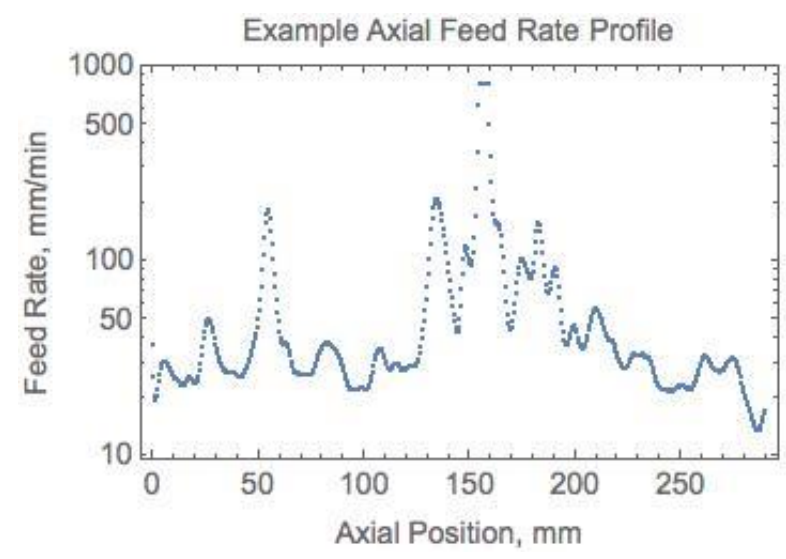

Figure 3. Feed rate profile calculated for the initial error map shown in figure. 4
A series of experiments were performed to develop and evaluate algorithms for applying the wear function to surface error correction. After trying various constrained fitting and deconvolution algorithms we found that a Richardson-Lucy deconvolution leads to the best overall polishing performance for simulated post-polished slope errors. Using metrology data from a sample to be polished and these algorithms, the software calculates the optimal tool path to reduce surface errors and generates the Zeekomachine control code corresponding to the path.

The model was used to polish, using a $40 \mathrm{~mm}$ bonnet, a NiP plated $140 \mathrm{~mm}$ diameter mandrel inherited from a previous project. A coordinate measuring machine was used for initial determination of the mandrel radii as function of axial position. Then, the optical interferometer was used to measure 36 axial profiles of the mandrel. Most of the initial slope errors of the mandrel are cylindrical, i.e. the surface slope profile are similar from meridian to meridian, so a feed rate map from one dimensional deconvolution of average axial error map was calculated and applied to all meridians. The Richardson-Lucy algorithm was used to calculate the federate profile that leads to smallest residual rms slope errors. An example of the calculated feed rate profile is shown in Figure 3. A few polishing runs were performed using the Zeeko machine and the polishing direction was altered from run to run in order to minimize the bonnet wear drift. A super-polishing run was performed on a traditional lap polishing machines to obtained required surface microroughness. An example of axial height profile before and after polishing experiments is shown in Figure 4. The respective power spectrum functions of the axial height errors calculated for the given meridional profile are shown in Figure 5. The surface slope errors were reduced from $6.3 \operatorname{arcsec}$ to $0.3 \mathrm{arcsec}$, which correspond to 1.2 arcsec HPD resolution (two-reflection equivalent, other sources of error, such as microroughness does not taken into account). The results of the deterministic polishing runs are summarized in Table 2. Further improvement 


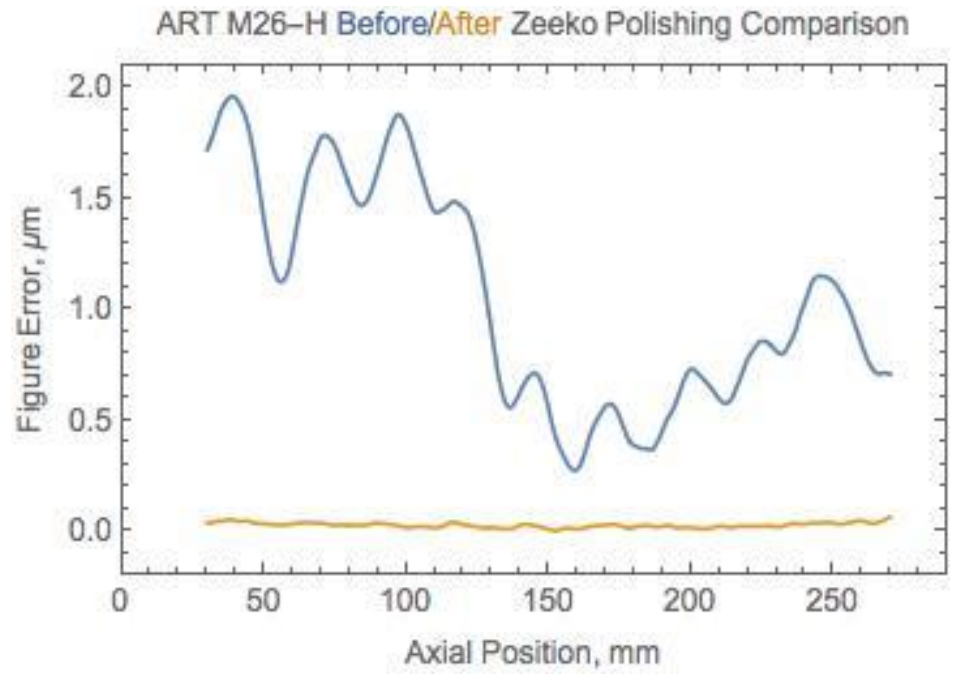

Figure 4. Surface height error profile of hyperbolic side of a NiP plated mandrel before (blue) and after (yellow) polishing using a $40 \mathrm{~mm}$ bonnet.

ART M26-H Before/After Zeeko Polishing Comparison

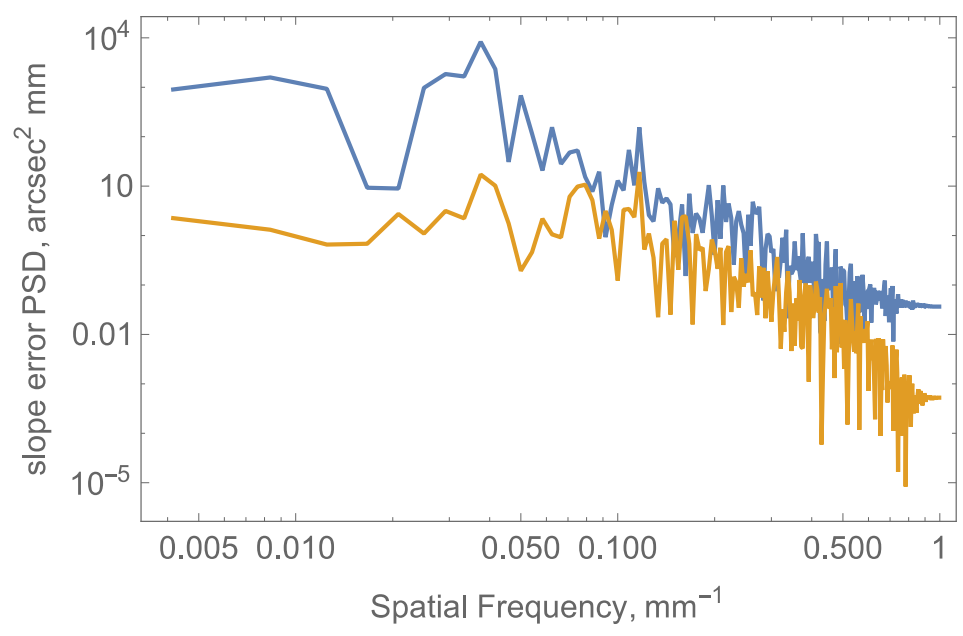

Figure 5. Power spectrum function calculated for surface height errors before and after polishing using a $40 \mathrm{~mm}$ bonnet. is possible using the Zeeko machine with a 20 mm bonnet, which produces a smaller footprint on the surface under polishing, so higher midfrequency errors can be attacked. However, the amplitude of the mid-frequency "bumps" on the axial profile are less than $30 \mathrm{~nm}$, well within the range that can be corrected by differential deposition for sub-arcsecond performance.

\subsection{Future work}

The next step in the process development will be transfer of the polishing technique to segmented and full shell mirrors. For this a NiPplated full shell, that can be later segmented, has been made from an aluminum tube with 0.25 meter inner diameter. The shell is shown in Figure 6. The segments are sufficiently thick that backing material is not needed for the polishing experiments. The tube is machined to the desired axial profile using a single point diamond turning machine and then coated with NiP. The tube is stress-relieved before and after each manufacturing step. Then, the plated tube will be diamond turned on both (inner and outer) surfaces again to provide the desired figure and to keep equal amount of nickel coating on each surface in order to and equalize any residual stresses in the coating. The full shell then will be cut into segments using axial channels that were cut with an EDM machine earlier during the fabrication process.

The fixtures to support the tube and the segments during diamond turning and polishing are designed to preserve the natural shape of the segments through all manufacturing processes. The stability of the tube form with and without the fixtures attached has been verified. The tube is made from extruded aluminum, so it is possible the stress reliving processes may not reduce stresses to zero; hence, the

Table 2. Results of deterministic polishing runs.

\begin{tabular}{|l|c|c|}
\hline Parameter & before & after \\
\hline Height error, $\mathrm{nm}$ rms & 500 & 10.7 \\
\hline Slope error (>2 mm), arc-seconds rms & 6.32 & 0.30 \\
\hline Low frequency (> 7 cm) slope error, arc-seconds rms & 2.66 & 0.09 \\
\hline Mid frequency (2 - 7 cm) slope error, arc-seconds rms & 5.73 & 0.29 \\
\hline
\end{tabular}


segments could flex after the tube is cut into pieces. Since the goal of this development is optimization of figuring and superpolishing processes for full-shell optics, the optical prescription can be changed to fit the new shape at this point. Note, segments would ultimately be made from beryllium alloys by low temperature additive technologies and sintering, or from Al-Si alloys by casting. In either case, they would pass through much more rigorous stress-relieving processes and thus the segments would be expected to have much greater dimensional stability. Any change in shape would be much less than the 100 micron of the NiP coating left after the diamond-turning step, which is available for fine figuring.

\section{CONCLUSIONS}

Fabrication technology to produce high-resolution x-ray

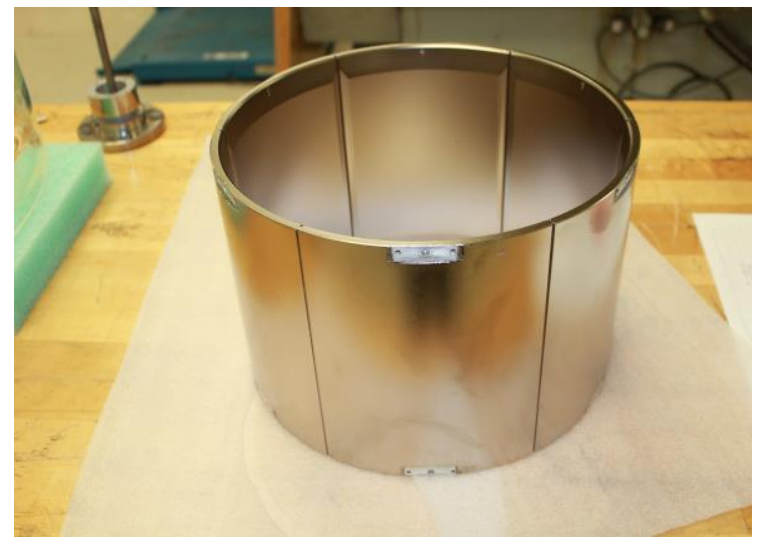

Figure 6. A NiP plated, segmented aluminum pipe with a conical inner figure fabricated for metrology, figuring and superpolishing experiments.

optics directly from thin metal substrates is under development at the MSFC. The use of metal substrates permits permits substituting single-point diamond turning in place of the grinding process used for fabrication of glass optics. After the mirror substrate is diamond turned, heat treated and machined to the final form and thickness, it will be plated with electroless-nickel alloy and diamond turned to optical prescription. Then the substrate is polished using the computer controlled polishing machine.

Two major challenges for this technology are to provide adequate support to the mirror substrates during all production steps and to figure the mirror without introducing additional mid-spatial frequency errors. In our previous paper we had presented the design of the fixtures aimed to support the mirrors during diamond turning, polishing and metrology [6]. The development of the polishing technique for direct fabrication of the $\mathrm{x}$-ray optics is in progress. The tool-path algorithm has been developed and verified using flat samples and a mandrel. For the mandrel the surface slope errors were reduced to 0.09 arc-seconds for the spatial wavelengths higher $7 \mathrm{~mm}$ (the limit for the $10 \mathrm{~mm}$ bonnet footprint used for experiments).

The next step in the process development is the transfer of the polishing technique to full shell and segmented x-ray mirrors. For this, a NiP plated, segmented aluminum tube with a conical inner figure has been fabricated. The tube is designed to be divided in 6 segments after it is diamond turned to the optical prescription. A fixture to support the segments during diamond turning, polishing and metrology has been designed and fabricated.

\section{REFERENCES}

[1] Zhang, W. W., M. P. Biskach, P. N. Blake, K. W. Chan, T. C. Evans, M. L. Hong, W. D. Jones et al. "Lightweight and high angular resolution x-ray optics for astronomical missions." In SPIE Optical Engineering+ Applications, pp. 81470K-81470K. International Society for Optics and Photonics, 2011.

[2] ] Ramsey, B.D. "Replicated nickel optics for hard x-ray region.” Experimental Astronomy, 20, pp. 85-92.

[3] R. E. Riveros, Linette D. Kolos ; James R. Mazzarella ; Kevin P. McKeon ; William W. Zhang., "Fabrication of high resolution and lightweight monocrystalline silicon x-ray mirrors," Proc. SPIE 9603, 96030W (2015)

[4] M. M. Civitani, O. Citterio, S. Campana, P. Conconi, E. Mattaini, G. Pareschi, G. Tagliaferri, G. Parodi, V. Burwitz, G. D. Hartner, J. Arnold, S. Schuler, H. Combrinck, R. Freeman, R. Morton, P. Simpson, D. Walker, " Thin glass shell oriented to wide field x-ray telescope ", Proc. SPIE 8443, 84430Q (2012).

[5] M. M. Civitani, O. Citterio, M. Ghigo, E. Mattaini, G. Pareschi, G. Parodi,,"Thin monolithic glass shells for futre high angular realution and large collecting area x-ray telescope”, Proc. SPIE 8884, 88841R (2013).

[6] M. Gubarev, B. Ramsey, J.J. Kolodziejczak, W. Jones, C. Griffith, J. Roche, W. S. Smith, C. Atkins, W. Arnold, "Direct Fabrication of full-shell x-ray optics", Proc. SPIE 9603, 96030V (2015) 
[7] J. A. Gaskin, M. C. Weisskopf, A.Vikhlinin, H. D. Tananbaum, S. R. Bandler, M. W. Bautz, D. N. Burrows, A. D. Falcone, F. A. Harrison, R. K. Heilmann, S. Heinz, R. C. Hopkins, C. A. Kilbourne, C. Kouveliotou, R. P. Kraft, A. V. Kravtsov, R. L. McEntaffer, P. Natarajan, S, L. O’Dell, R. Petre, A. F. Ptak, B. D. Ramsey, P. B. Reid, A. R. Schnell, D. A. Schwartz, L. K. Townsley, “The X-ray Surveyor Mission: A Concept Study”, Proc. SPIE 9601, 96010J (2015)

[8] http://www.zeeko.co.uk/site/tiki-index.php?page=IRP+Machine+Brochures

[9] K. Kilaru, C. Atkins, B.D. Ramsey, J.J. Kolodziejczak, T.M. Lis, M.V. Gubarev, S.L. O'Dell, J.A. Gaskin, D.M. Broadway, Progress in Differential Deposition for Improving the Figures of Full-Shell Astronomical Grazing Incidence X-Ray Optic. Proc Spie 9603, 96031F (2015)

[10] Michelle L. Delatte and Douglas L. Hibbard, "Fabrication of a large-aperture beryllium telescope for space application", Proc. SPIE 2542, Optomechanical and Precision Instrument Design, 258 (September 6, 1995).

[11] http://materion.com/ResourceCenter/NewsAndAnnouncements/Materion/MaterionMaterialsEnablingSpaceMissions .aspx.

[12] Ahmad, A., Engelhaupt, Feng, C., Hadaway, J., and Ye Li. "Design and fabrication of low cost light weight metal mirrors". In Proc. Manufacturing Process Development in Photonics Conf. Redstone Arsenal, AL, (1994). 